\title{
Biomarker SST2 in Adults with Transposition of the Great Arteries Palliated by Mustard Procedure
}

\author{
Haley Ferguson ${ }^{1}$, Hugo Martinez ${ }^{2}$, Melanie Pride ${ }^{2,3}$, Roger Hurwitz², Mark Payne ${ }^{2,3}$ \\ ${ }^{1}$ Indiana University School of Medicine, MS II; ${ }^{2}$ Indiana University School of Medicine, \\ Department of Pediatrics (Cardiology); ${ }^{3}$ Herman B Wells Center for Pediatric Research
}

\begin{abstract}
Background: Transposition of the great arteries (TGA) is a congenital heart defect (CHD) in which the aorta and pulmonary artery are transposed; it requires urgent surgical intervention. The Mustard procedure was an early surgery allowing survival into adulthood. However, this procedure is associated with long-term adverse effects including arrhythmias and heart failure (HF). A key factor impairing HF management in adults with $\mathrm{CHD}$ is lack of biomarkers to predict outcome. Soluble ST2 (sST2) is a protein secreted by myocytes in response to mechanical strain and fibrosis. No studies have focused specifically on SST2 in TGA individuals that underwent a Mustard procedure. In this study, we hypothesized adults with TGA palliated with a Mustard procedure would have higher levels of SST2 than healthy individuals, and this would correlate with functional class status.
\end{abstract}

Methods: We screened the Pediatric Cardiology database for D-TGA subjects and Mustard operation. Healthy controls were recruited from clinic and the community. Patients were matched by age to a control group of 21 healthy individuals. Severity of symptoms was assessed by NYHA functional classification. SST2 levels were obtained using Critical Diagnostics Presage ST2 Assay kit. Additionally, cTnl, BNP, lipid panel, insulin, glucose, and EKG and echocardiography (TGA) were obtained at IUSM clinical laboratories.

Results: We identified 45 patients with D-TGA and Mustard operation. 19 patients were included in analysis: 12 male and 7 female subjects aged 18 to 46 , mean of 34.9 years. In the Mustard group, 9 subjects were assigned to NYHA class II, and 9 to class III. The control group was asymptomatic. SST2 levels in the Mustard group were elevated in $53 \%$, while only $29 \%$ in the controls. Of the Mustard subjects with elevated sST2, $67 \%$ had elevated cTnl, $80 \%$ arrhythmias, $70 \%$ EKG T-wave abnormalities, and $80 \%$ took cardiac medications. $90 \%$ of these patients had low HDL, $40 \%$ high LDL, and $40 \%$ low insulin.

Conclusion: This study demonstrates patients with Mustard operation averaged higher SST2 levels than healthy subjects. Importantly, of the patients with elevated levels, there was a significant association of SST2 with biomarker abnormalities and clinical heart failure signs. This suggests in patients with D-TGA palliated with Mustard procedure, SST2 may add predictive value to cardiac related morbidity and mortality. 